\title{
Novel dural incision and closure procedure for preventing postoperative cerebrospinal fluid leakage during the surgical removal of dumbbell-shaped spinal tumors: technical note
}

\author{
Kiyoshi Ito, MD, Tatsuro Aoyama, MD, Takuya Nakamura, MD, Yoshiki Hanaoka, MD, \\ Tetsuyoshi Horiuchi, MD, and Kazuhiro Hongo, MD \\ Department of Neurosurgery, Shinshu University School of Medicine, Matsumoto, Japan
}

The authors report on a new method for removing dumbbell-shaped spinal tumors that avoids the risk of postoperative cerebrospinal fluid (CSF) leakage. Adequate visualization of the intra- and extradural components of the tumor is achieved with the use of separate dural incisions. First, the dura mater is opened along the dural theca to provide adequate visualization of the intradural portion of the mass; then, a second incision is made along the nerve root to remove the extradural component. Meticulous suturing is essential in intradural lesion cases; however, the dura mater is usually thin and fragile in such cases. During suturing with a needle and thread, the dura mater can become lacerated proximal to the needle holes and result in CSF leakage. In the authors' technique, instead of using a needle and thread, nonpenetrating vascular clips were used to close the dural incisions. When operating on dumbbell-shaped spinal tumors, the authors found that the "separate-dural-incision method" was preferable to the conventional T-shaped dural incision method because no dural defects occurred after the intradural procedure and meticulous dural closure with vascular clips was achieved. The authors conclude that the novel separate-dural-incision method for removing dumbbell-shaped tumors and the use of nonpenetrating vascular clips permits reliable dural closure, prevents postoperative CSF leakage, and promises good postoperative clinical results.

http://thejns.org/doi/abs/10.3171/2016.3.SPINE151538

KEY WORDS dumbbell-shaped tumor; dural incision; postoperative cerebrospinal leakage; spinal cord tumor; surgical approach; technique

$\mathrm{P}$ OSTOPERATIVE cerebrospinal fluid (CSF) leakage is one complication encountered when performing spinal surgery. It is important to prevent this complication, especially in surgery for spinal intradural lesions, as patients with persistent CSF leakage are at risk for the development of meningitis, arachnoiditis, and pseudomeningoceles.

Dural incision and subsequent suturing affect the postoperative clinical result, particularly in patients with dumbbell-shaped spinal tumors. Thus, various surgical techniques for removing dumbbell-shaped tumors have been described. ${ }^{1,2,9}$ Since these lesions extend into the intraand extradural spaces through the dura mater, surgery for this type of tumor often utilizes a T-shaped dural incision, which is made parallel to the spinal canal and nerve root to provide good visualization of the tumor. In most cases, the T-shaped dural incision is placed above the tumor mass (Fig. 1A), and after the tumor is removed, the incisions are sutured with nylon or expanded polytetrafluoroethylene sutures; however, dural laceration and dural defects along with insufficient closure can occur during this process and lead to postoperative CSF leakage (Fig. 1B and C).

To avoid CSF leakage and subsequent adverse events in intradural lesion cases, meticulous closure is essential; however, the dura mater is usually thin and fragile in these cases. ${ }^{5,11}$ Additionally, during intradural procedures, the dura mater often becomes dry and shrinks, making it difficult to suture the T-shaped incision precisely without defect or CSF leakage. For this reason, watertight dural suturing is more difficult in spinal intradural procedures than in intracranial procedures.

The cranial dura mater is composed of 2 histologically 


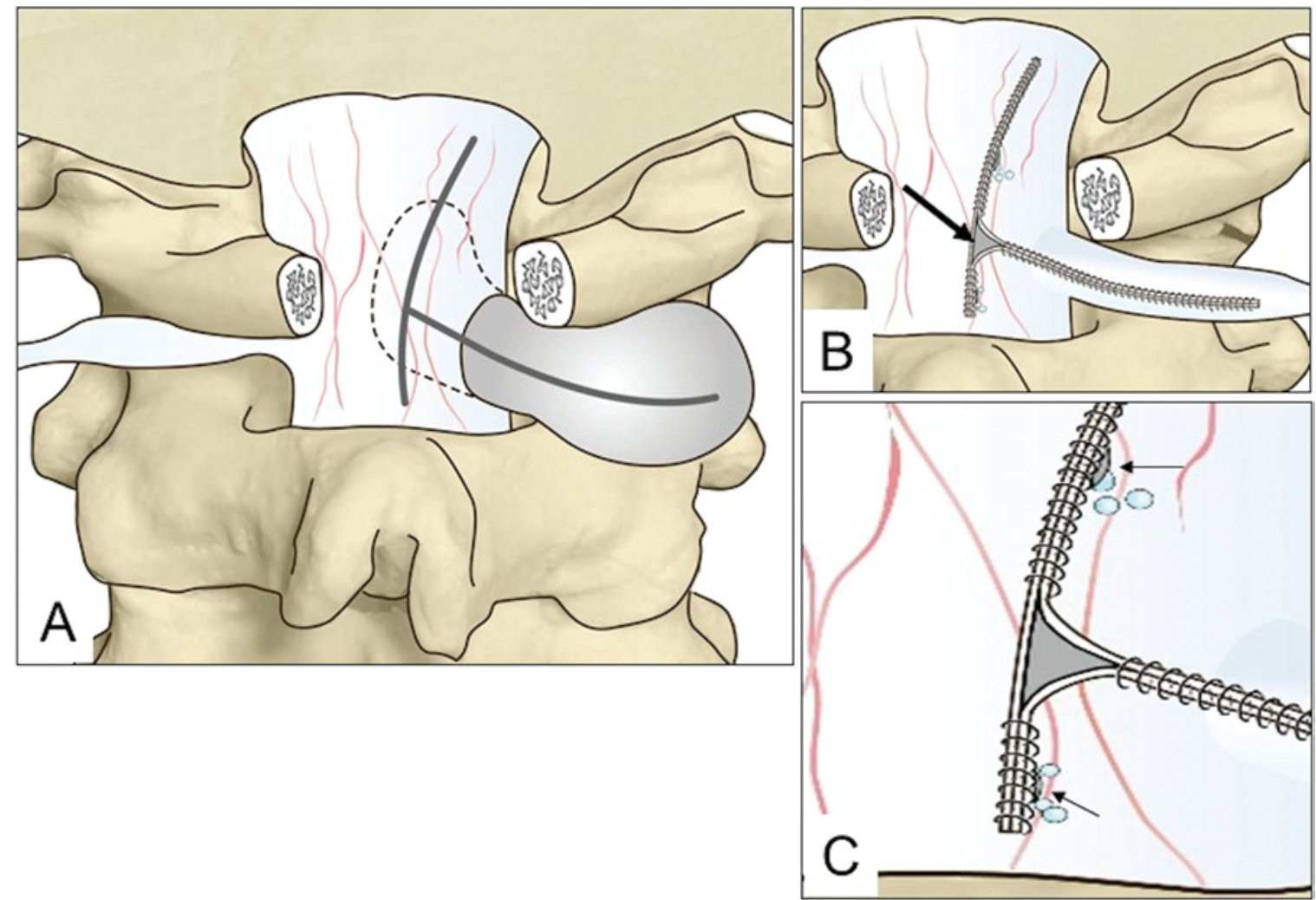

FIG. 1. Schematic drawing showing the conventional T-shaped dural incision method with needle and thread suturing. A: The spinal dura mater is incised in a T-shaped fashion over the affected region. In doing so, it is possible to remove the entirety of a tumor that extends into the intra- and extradural regions using a single dural incision. B: After performing the intradural procedure and suturing the dural incisions, a dural defect is often created in the area where the incisions cross (arrow). C: Magnified view of the crossing point showing the dural defect from a T-shaped dural incision. Dural lacerations (small arrows) are created proximal to the needle holes and spinal fluid can potentially leak from these needle holes and dural lacerations. Copyright Kyoshi Ito. Published with permission.

designated layers, namely the periosteal and meningeal layers, and each layer contains differently patterned collagen fibers. In contrast, the spinal dura mater is composed of a single meningeal layer, with collagen fibers oriented in a longitudinal direction. ${ }^{5}$ Because the collagen fibers of the spinal dura mater are oriented in the same direction, the dura mater can tear easily during surgical suturing with a needle and thread. Furthermore, the delicate structure of the spinal dura mater increases the possibility of lacerations proximal to the needle holes. CSF leakage can occur from these suture needle holes and dural lacerations. Therefore, new surgical approaches are needed to decrease the likelihood of dural lacerations during closure and subsequent CSF leakage. Here, we report on our experience with an alternative approach, referred to as the separate-dural-incision method, that utilizes nonpenetrating vascular clips for incision closure following the excision of a dumbbell-shaped spinal tumor. We furthermore discuss the efficacy and technical advantages of using the separate-dural-incision method as compared with the conventional T-shaped dural incision method.

\section{Methods}

This study was approved by the institutional review board of Shinshu University School of Medicine.

\section{Patients}

Informed consent was obtained prior to surgery from all patients who participated in this study. The participants included 2 patients with an Eden Type I C-2 dumbbellshaped schwannoma and 1 patient with an Eden Type I S-2 dumbbell-shaped schwannoma ${ }^{3}$ who underwent total tumor resection between April and November of 2015. Patient data and tumor characteristics are listed in Table 1 . The patients presented with motor and sensory disturbances that interfered with daily life and were treated with the surgical techniques described below.

\section{Surgical Technique}

In this section, we briefly describe the surgical procedures we used for removing the dumbbell-shaped tumors. The patient was placed in a prone position under general

TABLE 1. Patient data and tumor characteristics

\begin{tabular}{cccccc}
\hline $\begin{array}{c}\text { Case } \\
\text { No. }\end{array}$ & $\begin{array}{c}\text { Age (yrs), } \\
\text { Sex }\end{array}$ & $\begin{array}{c}\text { Tumor } \\
\text { Origin }\end{array}$ & Side & $\begin{array}{c}\text { Eden } \\
\text { Classification }\end{array}$ & $\begin{array}{c}\text { Amount of } \\
\text { Hemorrhage (g) }\end{array}$ \\
\hline 1 & $63, \mathrm{M}$ & C-2 & Lt & Type I & 50 \\
\hline 2 & 58, F & C-2 & Rt & Type I & 100 \\
\hline 3 & 72, M & S-2 & Rt & Type I & 70 \\
\hline
\end{tabular}


anesthesia. A midline skin incision was made, and then midline splitting of the affected spinous processes was performed. The paravertebral fascia and muscles were dissected to preserve the posterior tension band, including the nuchal, supraspinous, and interspinous ligaments, and the spinous processes. Laminectomy was performed and the dural sac was exposed. Next, the dura mater was opened with 2 separate incisions: the first incision was made along the dural theca to provide adequate visualization of the intradural portion of the tumor mass, and the second incision was made along the nerve root to allow visualization of the extradural portion of the tumor mass (Fig. 2A). Surgery was conducted with the utmost care so as to avoid connecting the separate dural incisions. After removal of the intradural portion and sectioning of the tumor through the incision along the dural theca, the extradural component was pulled through the incision along the nerve root and removed (Fig. 2B). The dura was closed with nonpenetrating titanium clips (vascular clip system [VCS] clips; LeMaitre Vascular Inc.) (Fig. 2C), and each incision was covered with absorbable polyglycolic acid mesh and fibrin glue. After closure of the dura, the Valsalva maneuver was performed to check for CSF leakage. The wound was well irrigated and closed in layers.

\section{Postoperative Evaluations}

To determine the effectiveness of the surgical method, we used postoperative visual observations, neurological examinations, and MRI. These measures allowed us to evaluate the patients' neurological symptoms after surgery and establish the completeness of tumor resection. Postoperative neuroimages were obtained with a 1.5-T MRI unit (Optima MR450w, GE Medical Systems), with both T1- and T2-weighted sequences used to evaluate postoperative CSF leakage. All images were obtained 1 week after surgery.

\section{Results}

Linear dural incisions were meticulously closed with nonpenetrating vascular clips according to the separatedural-incision method and did not produce dural defects. The Valsalva maneuver revealed no intraoperative CSF leakage in any of the cases. Dumbbell-shaped tumors were completely removed with the aforementioned method. Postoperatively, no patient showed CSF leakage on MR images.

\section{Illustrative Case}

This 73-year-old man presented with a 1-year history of low-back pain without motor weakness (Table 1). He visited a local clinic where gadolinium-enhanced T1-weighted MRI showed a right-lateralized S-2 dumbbell-shaped neurinoma, which was classified as an Eden Type I tumor
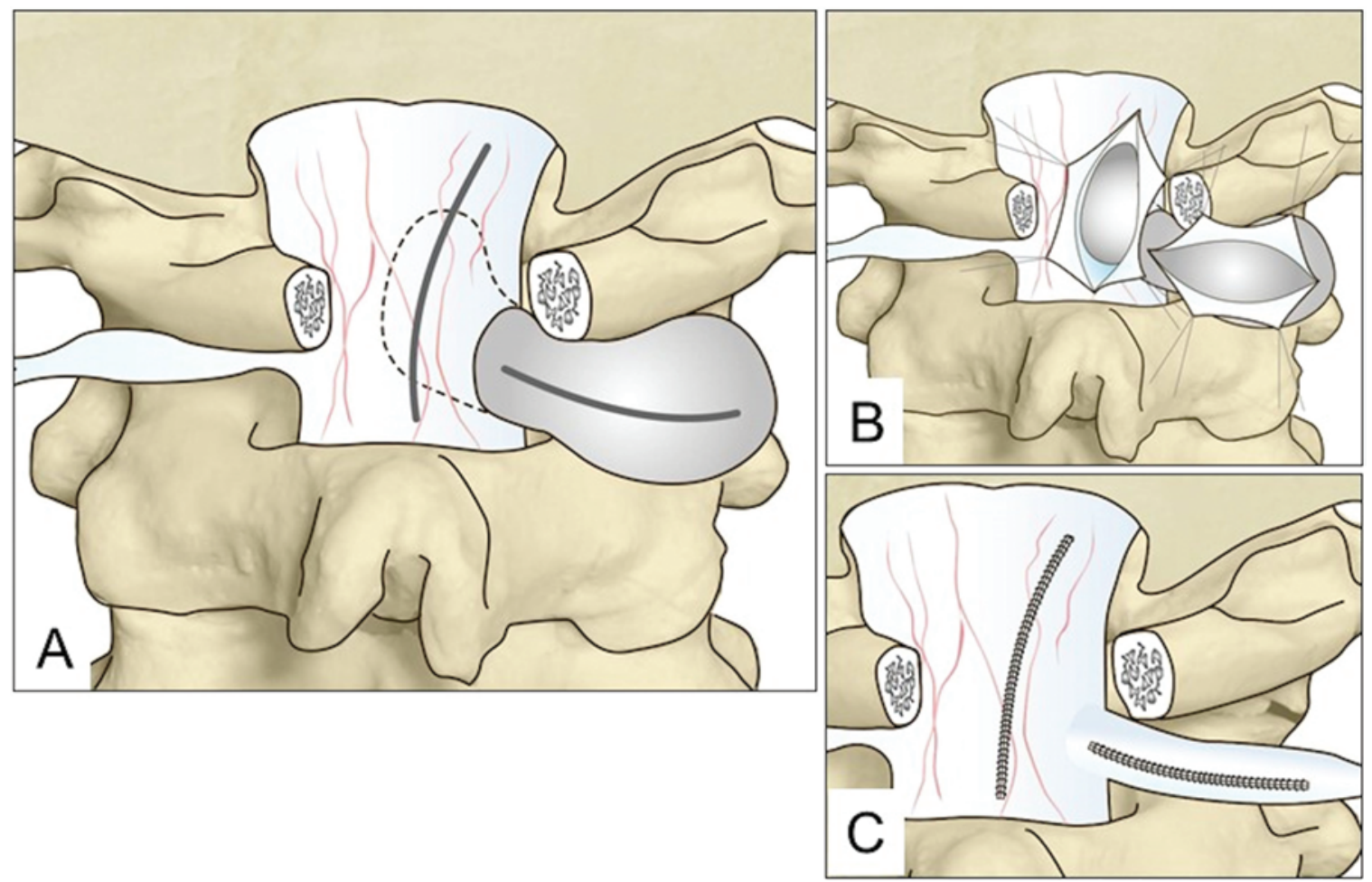

FIG. 2. Schematic drawing showing the separate-dural-incision method using nonpenetrating titanium vascular clips for closure. A: The spinal dura mater is incised in a separate fashion, in which one incision is made along the dural theca to provide adequate visualization of the intradural portion of the tumor mass, and a second incision is made along the nerve root to allow visualization of the extradural portion of the tumor mass. The surgical procedure is performed with special care so as not to connect the dural incisions. B: After the intradural portion is removed and the tumor is sectioned through the dural incision along the dural theca, the extradural component is pulled through the incision along the nerve root and removed. C: The dura is closed with nonpenetrating titanium clips. No dural defect or laceration is created. Copyright Kyoshi Ito. Published with permission. 
(Fig. 3A-D). He was referred to our hospital for further examination and surgical intervention. Upon admission to our hospital, a neurological examination showed no motor weakness, but the patient reported severe pain in his right lower back. Neuroimages showed a strongly enhancing mass compressing the cauda equina. The tumor was surgically removed using the separate-dural-incision method. First, the intradural pathology was removed (Fig. 4A); the resected stumps are shown in Fig. 4B. Second, the extradural lesion was removed (Fig. 4C and D). A linear incision was made along the nerve sheath, and the extradural component of the tumor was removed and the edge of the intradural lesion was pulled out. After closure of the dura mater, the Valsalva maneuver was performed to confirm the absence of intraoperative CSF leakage. Postoperatively, the patient's severe pain was relieved. Postoperative MRI showed complete removal of the tumor. Procedurerelated complications, such as instability or CSF leakage, were not observed (Fig. 3E).

\section{Discussion}

Surgery for intradural tumors, especially dumbbellshaped intradural tumors, often utilizes a T-shaped dural incision that is made parallel to the spinal canal and nerve root to provide good visualization of the tumor. However, it is difficult to suture the dura mater in a T-shaped fashion precisely and without defect or postoperative CSF leak- age. Since the prevention of postoperative CSF leakage is essential for ensuring a good outcome, new surgical approaches that can avoid CSF leakage are needed. We developed a novel method for the removal of dumbbellshaped tumors that utilizes separate dural incisions and nonpenetrating titanium vascular clips. We found that this method did not cause any postoperative CSF leakage and improved patient outcomes.

Authors of previous studies have proposed the use of several different sealing materials, such as fibrin glue and an absorbable polyglycolic acid mesh, as adjuncts to dural sutures to reduce the potential for adverse events. ${ }^{4,6,8,10}$ However, CSF leakage cannot always be prevented with the use of these adjunctive materials. Here, we found that compared with the T-shaped dural incision method, the separate-dural-incision method enabled the surgeon to reduce the likelihood of CSF leakage with the use of a simpler and more reliable dural closure method and linear incisions. Instead of using several different sutures, such as nylon or expanded polytetrafluoroethylene sutures, we used nonpenetrating vascular clips to close the linear dural incisions. Needle and thread suturing methods can produce dural lacerations proximal to the needle holes, and spinal fluid may leak from these needle holes and lacerations. Therefore, nonpenetrating titanium vascular clips, which do not create any suture holes, are ideal for closing dural incisions.,
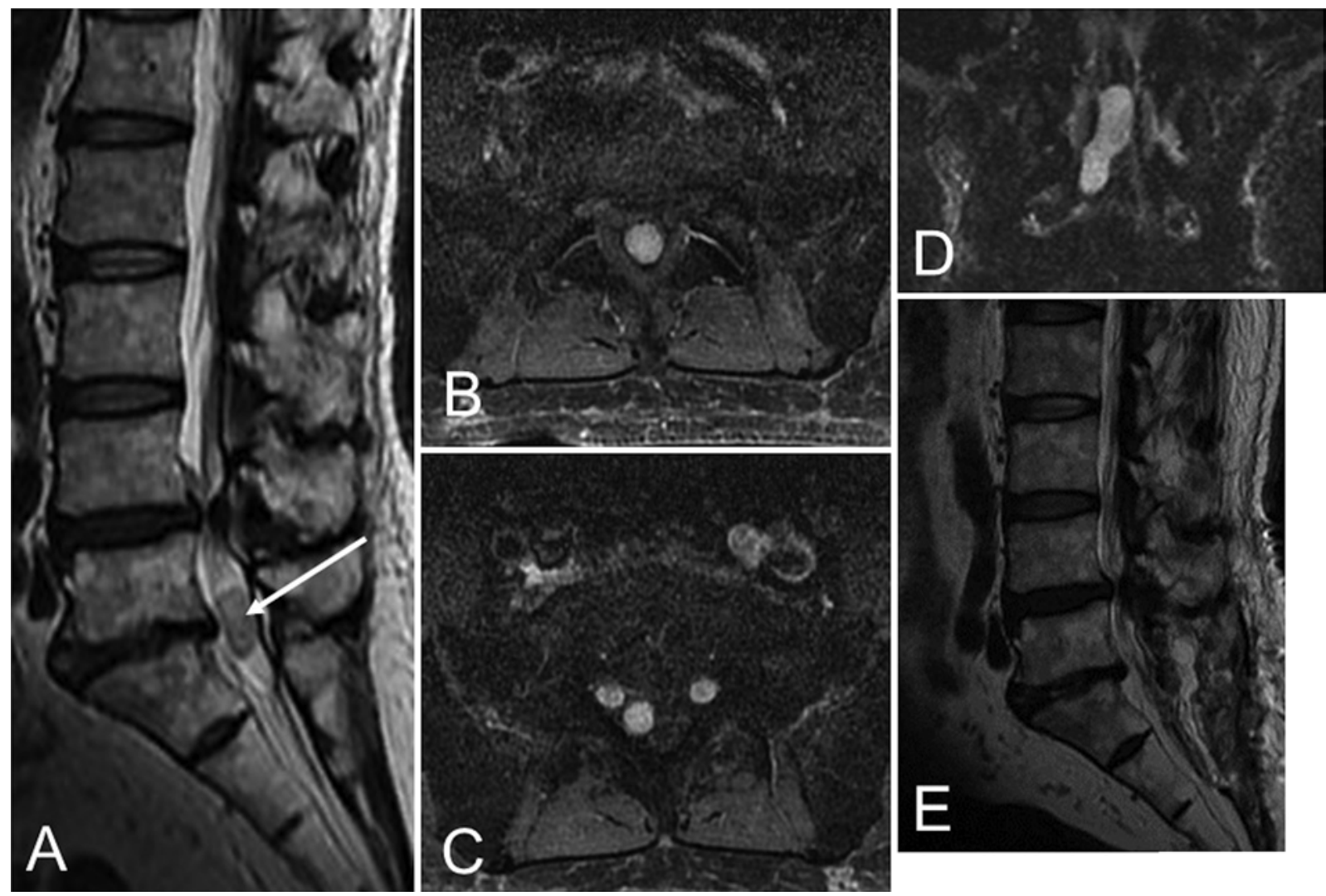

FIG. 3. Illustrative case involving a 73-year-old man with an S-2 neurinoma. A: Preoperative T2-weighted sagittal MR image showing a round mass at L5-S1 (arrow). B-D: Preoperative axial and coronal T1-weighted MR images obtained after administration of gadolinium showing a right-lateralized S-2 dumbbell-shaped neurinoma (Eden Type I). E: Postoperative T2-weighted sagittal MR image showing no CSF leakage. 

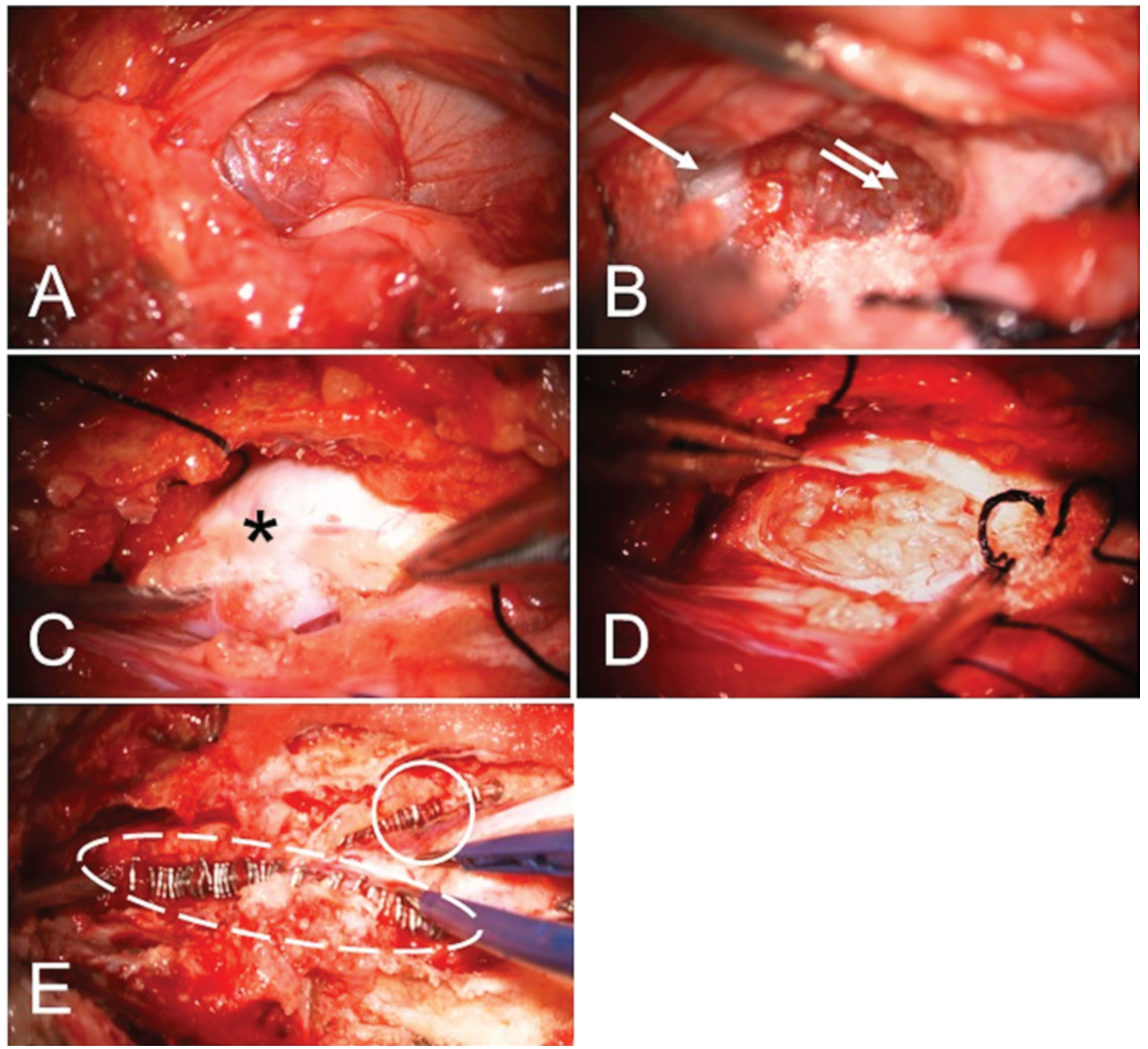

FIG. 4. Intraoperative photographs from illustrative case showing the separate-dural-incision method with nonpenetrating vascular clips. A: After incision of the thecal sac the intradural pathology becomes visible. B: The intact right S-2 nerve root becomes visible after removal of the intradural mass (single arrow). The resected stumps of the removed intradural portion are shown (double arrows). C: The right S-2 nerve root is dissected (asterisk). D: The extradural lesion is removed through the linear incision along the nerve sheath. After removal of the extradural component of the tumor, the edge of the intradural lesion is pulled out. E: After the dura mater is closed, the Valsalva maneuver is performed to confirm the absence of intraoperative CSF leakage. The brokenline oval indicates the incision through which the intradural lesion was removed; the small circle indicates the incision through which the extradural lesion was removed.

Surgeons should be aware that the interval between the 2 incisions can potentially interfere with tumor visualization. However, the present method utilizes 2 simple incisions that enable the surgeon to perform reliable closure to reduce postoperative CSF leakage and yield a good surgical result. There are several limitations to our study that should be noted, including a small sample size of 3 patients and a comparatively short follow-up period. The cases that we have reported warrant additional follow-up in the future to better inform the efficacy of our novel procedure. However, we believe that the usefulness of our approach for the prevention of postoperative CSF leakage is valid on the basis of theory and our experience

\section{Conclusions}

Based on a limited number of observations, we conclude that the separate-dural-incision method and the use of nonpenetrating vascular clips are useful for removing dumbbell-shaped spinal tumors. With this approach, suture-related dural defects and lacerations can be avoided and postoperative CSF leakage can be prevented.

\section{Acknowledgments}

We thank Mr. S. Ono for his technical support. This work was supported by the JSPS KAKENHI, grant no. 15K10356.

\section{References}

1. Asazuma T, Toyama Y, Maruiwa H, Fujimura Y, Hirabayashi K: Surgical strategy for cervical dumbbell tumors based on a three-dimensional classification. Spine (Phila Pa 1976) 29:E10-E14, 2004

2. Conti P, Pansini G, Mouchaty H, Capuano C, Conti R: Spinal neurinomas: retrospective analysis and long-term outcome of 179 consecutively operated cases and review of the literature. Surg Neurol 61:34-44, 2004 
3. Eden K: The dumb-bell tumours of the spine. Br J Surg 28:549-570, 1941

4. Hida K, Yamaguchi S, Seki T, Yano S, Akino M, Terasaka $\mathrm{S}$, et al: Nonsuture dural repair using polyglycolic acid mesh and fibrin glue: clinical application to spinal surgery. Surg Neurol 65:136-143, 2006

5. Ito K, Aoyama T, Horiuchi T, Hongo K: Utility of nonpenetrating titanium clips for dural closure during spinal surgery to prevent postoperative cerebrospinal fluid leakage. J Neurosurg Spine 23:812-819, 2015

6. Ito K, Ishizaka S, Sasaki T, Miyahara T, Horiuchi T, Sakai K, et al: Safe and minimally invasive laminoplastic laminotomy using an ultrasonic bone curette for spinal surgery: technical note. Surg Neurol 72:470-475, 2009

7. Kaufman BA, Matthews AE, Zwienenberg-Lee M, Lew SM: Spinal dural closure with nonpenetrating titanium clips in pediatric neurosurgery. J Neurosurg Pediatr 6:359-363, 2010

8. Nagata K, Kawamoto S, Sashida J, Abe T, Mukasa A, Imaizumi Y: Mesh-and-glue technique to prevent leakage of cerebrospinal fluid after implantation of expanded polytetrafluoroethylene dura substitute-technical note. Neurol Med Chir (Tokyo) 39:316-319, 1999

9. Ozawa H, Kokubun S, Aizawa T, Hoshikawa T, Kawahara C: Spinal dumbbell tumors: an analysis of a series of 118 cases. J Neurosurg Spine 7:587-593, 2007
10. Shaffrey CI, Spotnitz WD, Shaffrey ME, Jane JA: Neurosurgical applications of fibrin glue: augmentation of dural closure in 134 patients. Neurosurgery 26:207-210, 1990

11. Standring S: Gray's Anatomy: The Anatomical Basis of Clinical Practice, ed 40. Edinburgh: Churchill Livingstone/ Elsevier, 2008

\section{Disclosures}

The authors report no conflict of interest concerning the materials or methods used in this study or the findings specified in this paper.

\section{Author Contributions}

Conception and design: Ito, Horiuchi. Acquisition of data: all authors. Analysis and interpretation of data: Ito, Horiuchi, Hongo. Drafting the article: Ito. Critically revising the article: Ito, Hongo. Reviewed submitted version of manuscript: Ito, Aoyama, Nakamura, Hanaoka, Horiuchi. Approved the final version of the manuscript on behalf of all authors: Ito. Study supervision: Hongo.

\section{Correspondence}

Kiyoshi Ito, Department of Neurosurgery, Shinshu University School of Medicine, 3-1-1 Asahi, Matsumoto 390-8621, Japan. email: kitoh@shinshu-u.ac.jp. 